\title{
Emprego dos métodos Utilité Additive e Utilité Additive - CRiteria na avaliação de imóveis: um estudo de caso
}

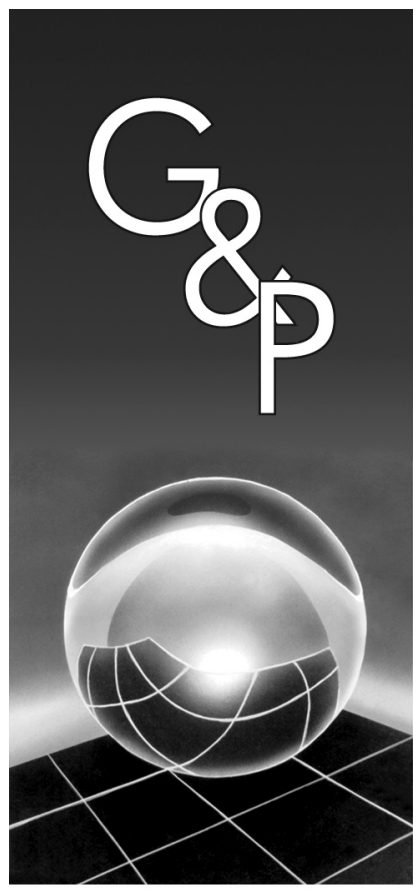

Luís Alberto Duncan Rangel Luiz Flávio Autran Monteiro Gomes

\begin{abstract}
Resumo
Este artigo apresenta uma aplicação do método Utilité Additive - UTA e de sua variante Utilité Additive - CRiteria - UTA-CR à determinação das funções de utilidade para se abordar o problema da avaliação de imóveis residenciais. Ambos os métodos pertencem à Escola Americana de Apoio Multicritério à Decisão e visam determinar funções de utilidades dos critérios presentes na análise, baseando-se em informações a priori dos decisores. Pelo emprego dos modelos matemáticos referentes a cada uma das metodologias, verificam-se as vantagens do método UTA-CR. Observa-se ainda que o método UTA-CR faz uso das preferências dos decisores em relação ao conjunto de critérios, obtendo funções de utilidades o mais próximo possível das preferências dos decisores.
\end{abstract}

Palavras-chave: Método UTA. Método UTA-CR. Método TODIM. Apoio multicritério à decisão. Função de utilidade multiatributo. Mercado imobiliário residencial.

\section{Introdução}

O método UTA (Utilité Additive) (JACQUET-LAGRÈZE; SISKOS, 1982) e sua variante UTA-CR (Utilité Additive CRiteria) (RANGEL, 2002) foram empregados nesta pesquisa com a finalidade de avaliar um subconjunto selecionado de imóveis residenciais que estão disponíveis para serem alugados no município de Volta Redondo, localizado ao sul do Estado do Rio de Janeiro. O emprego desses dois métodos na pesquisa objetivou principalmente a obtenção de funções de utilidades dos critérios utilizados na análise dos imóveis residenciais.

As imobiliárias, empregando em uma análise de imóveis com preços de aluguéis já definidos, isto é, já em carteira, com imóveis com preços de aluguéis a serem estimados, determinam os valores de referência dos aluguéis dos imóveis sem preços definidos. Este valor de referência pode ser obtido pela ordenação de todos os imóveis, com preços já definidos e a definir, utilizando as funções de utilidades dos critérios determinadas pelo método UTA.

O método UTA, para ser empregado, necessita de uma informação a priori dos decisores: a ordenação das alternativas ou de um subconjunto destas presentes no processo. Utiliza-se esta ordenação fornecida pelos decisores como restrição de um problema de programação linear (PPL). Este PPL tem por objetivo minimizar o somatório dos erros associados à ordenação de cada alternativa, isto é, o valor da utilidade global de cada alternativa proposta pelos decisores.

O método UTA-CR, uma variante do método UTA, visa obter as funções de utilidades o mais próximo possível das preferências dos decisores. Para obter essas novas funções de utilidades dos critérios, solicita-se aos decisores que expressem as suas preferências em relação ao conjunto de critérios e não em relação às alternativas, como ocorre quando se usa o método UTA original. Emprega-se então um método de ordenação do Apoio Multicritério à Decisão da problemática $\mathrm{P} \delta$, de ordenação das alternativas. Esta informação é utilizada na implementação do método UTA-CR, do mesmo modo que foi feito no método UTA original. Para obter funções de utilidades o mais próximo possível das preferências dos decisores, desenvolveu-se um novo modelo matemático que apresenta uma nova função objetivo e novas restrições.

No estudo apresentado neste artigo, ao invés de se solicitar a um especialista uma ordenação subjetiva dos imóveis presentes no processo para servir como dado de entrada na implementação do método UTA e da sua variante UTA-CR, empregou-se a ordenação dos imóveis obtida com o emprego do método TODIM (GOMES et al., 2004; GOMES; 
LIMA, 1992a; GOMES; LIMA, 1992b; GOMES, 2007), divulgada no artigo de Rangel et al., 2006.

A avaliação de imóveis é um assunto amplo e este estudo de caso realizado só foi possível com a colaboração de algumas imobiliárias que operam em Volta Redonda, além da participação de um especialista que emite parecer para Perícias Judiciais. Existem normas da Associação Brasileira de Normas Técnicas abordando a área de avaliações de imóveis (ABNT, 2004). Este estudo de caso foi realizado tomando-se como base as informações fornecidas pelos decisores que, neste caso, são as imobiliárias e o especialista. Os métodos de cálculo de aluguel de imóveis normalmente utilizados são (GUIMARÃES NETO, 1992; PESSOA, 1992):

- Correção Monetária, que consiste na aplicação de índices monetários oficiais ao aluguel inicial do contrato. O resultado assim obtido deve ser encarado apenas como um elemento afirmativo na avaliação, uma vez que o seu cálculo não envolve qualquer consideração quanto a características específicas do imóvel e considerações de mercado, bem como não corrige alguma eventual falha que haja ocorrido na fixação do aluguel inicial.

- Método Comparativo, que consiste na obtenção do aluguel por meio da análise estatística de uma amostra de valores locativos de níveis assemelháveis e localizados na mesma região geoeconômica, obtidos por pesquisa e devidamente homogeneizados. A precisão do resultado é função direta da qualidade e da confiabilidade da amostra selecionada, cabendo ao avaliador a análise prévia dessa confiabilidade para decidir sobre a conveniência da utilização do método.

- Método da Rentabilidade, que consiste na obtenção do aluguel por meio da aplicação de uma taxa de rentabilidade ao capital representado pelo valor do imóvel.

Este estudo de caso buscou determinar um método alternativo para estabelecer uma ordenação dos imóveis residenciais para serem alugados em termos de preço de aluguel, baseando esta avaliação em seis critérios selecionados pelos decisores que aqui são as imobiliárias e um especialista da área. Além da ordenação das alternativas de decisão, os métodos empregados neste estudo de caso, métodos UTA e UTA-CR, determinam as funções de utilidades dos critérios presentes na análise.

As funções de utilidades empregadas nestes dois métodos de Apoio Multicritério à Decisão são funções que descrevem as preferências do decisor ou de um grupo de decisores, em relação aos critérios de decisão presentes na análise. Desta forma, as curvas destas funções de utilidade podem ser utilizadas como referência pelos decisores para avaliar, por exemplo, algum outro imóvel que não estava previamente na análise, considerando os mesmos critérios do problema de decisão.

Realizaram-se as implementações dos dois métodos com a finalidade de verificar as diferenças entre as funções de utilidades obtidas com o método UTA original e com a sua variante UTA-CR. Apresentam-se e discutem-se os resultados das duas implementações na seção três deste artigo.

\section{Apresentação dos métodos Utilité Additive e Utilité Additive - CRiteria}

\subsection{Método Utilité Additive}

O método UTA (JACQUET-LAGRĖZE; SISKOS, 1982) possui as mesmas bases axiomáticas subjacentes à Teoria da Utilidade Multiatributo (MAUT) e é empregado para determinar as funções de utilidades dos critérios (KEENEY; RAIFFA, 1993; BELTON; STEWART, 2002).

Este método emprega a programação linear para determinar os valores das variáveis e, assim, serem obtidas as funções de utilidades. A função objetivo deste modelo busca minimizar o somatório dos erros associados ao valor global das alternativas, de modo a respeitar as preferências propostas a priori. Assim, o problema de regressão ordinal que é tratado pelo método UTA é o seguinte:

Tendo uma estrutura de preferência de pré-ordem R, $(\succ, \sim)$ com " $\succ$ " significando preferência estrita e " $\sim$ " a indiferença em um conjunto de alternativas ou ações, o ajuste da função de utilidade aditiva baseado em critérios múltiplos é obtido de tal modo que a estrutura de preferência resultante seja tão consistente quanto possível com a estrutura inicial (JACQUET-LAGRÈZE; SISKOS, 1982), proposta a priori pelos decisores. Os dados para implementar este método são a ordenação a priori dos decisores e a matriz de avaliação, isto é, o desempenho das alternativas em relação aos critérios.

Em um problema de Apoio Multicritério à Decisão, o conjunto de alternativas viáveis é avaliado por um conjunto de critérios definidos pelos avaliadores, com a finalidade de analisar as características das alternativas. No modelo aditivo, quando se emprega somente um critério, a preferência entre as alternativas, dentre o conjunto de alternativas $A$, pode ser explicitada como segue (Equações 1 e 2);

$$
\begin{gathered}
a \succ b \Leftrightarrow g_{i}(a)>g_{i}(b), \text { para } i=1, \ldots, n, \\
a \sim b \Leftrightarrow g_{i}(a)=g_{i}(b), \text { para } i=1, \ldots, n,
\end{gathered}
$$

que significa que este critério define no conjunto $A$ uma relação de ordem $(\succ, \sim)$ das alternativas.

Considere-se o conjunto de alternativas $A$, que é avaliado por um conjunto de critérios $\mathrm{g}=\left(g_{1}, g_{2}, g_{3}, \ldots, g_{n}\right)$ sendo $n$ o número de critérios e $g_{1}$ o desempenho no critério $i$. 
Denomina-se a agregação de todos os critérios em um único critério de função de utilidade multiatributo e é representada como $U(g)=U\left(g_{1}, g_{2}, g_{3}, \ldots g_{n}\right)$. Chama-se de $P$ a relação de preferência estrita e de $I$ a relação de indiferença (ROY; BOUYSSOU, 1993; VINCKE, 1989) entre duas alternativas, respectivamente.

Se $g(a)=\left[g_{1}(a), g_{2}(a), g_{3}(a), \ldots, g_{\mathrm{n}}(\mathrm{a})\right]$ é a avaliação multicritério de uma alternativa $a$, então se observam as seguintes propriedades da função de utilidade multiatributo $U$, em relação ao conjunto de alternativas $A$ :

$$
\begin{gathered}
U[g(a)]>U[g(b)] \Leftrightarrow a P b \\
U[g(a)]=U[g(b)] \Leftrightarrow a I b
\end{gathered}
$$

e a relação $R=P \cup I$ define uma pré-ordem das alternativas.

A função de utilidade é aditiva quando tem a forma abaixo (Equação 5):

$$
U[g]=\sum_{i=1}^{n} u_{i}\left(g_{i}\right)
$$

em que cada $u_{i}\left(g_{i}\right)$ é a utilidade marginal do desempenho $g_{i}$ no critério $i$. Uma hipótese fundamental que é preciso respeitar quando se aplica uma função de utilidade aditiva é a condição de independência mútua dos critérios em função das preferências (KEENEY; RAIFFA, 1993; ZOPOUNIDIS; DIMITRAS, 1998).

Considere $g_{i^{*}}$ e $g_{i}{ }^{*}$ o limite inferior e superior de cada critério $i$ presente num problema decisório. Assumindo um não decréscimo das preferências em cada critério, então as utilidades marginais $u_{i}$ são funções monótonas crescentes ou decrescentes. Portanto, podem-se normalizar as funções de utilidade dentro do intervalo [0,1], obtendo-se:

$$
\begin{gathered}
\sum_{i=1}^{n} u_{i}\left(g_{i}{ }^{*}\right)=1 \\
\text { e } u_{i}\left(g_{i^{*}}\right)=0 \text { para todo } \mathrm{i}=1,2, \ldots, \mathrm{n} .
\end{gathered}
$$

Empregam-se as Equações (6) e (7) na normalização das funções de utilidade. Faz-se uso da Equação (6) na normalização dos valores máximos de cada critério, limitando que este somatório dos valores máximos de cada critério seja igual a uma unidade. Já a Equação (7) por sua vez atribui o valor zero ao valor inicial de cada critério. Desta forma, as funções de utilidade que serão determinadas para cada critério apresentam um valor inicial igual a zero e o valor máximo a ser determinado por meio de programação linear.

O método UTA emprega a programação linear para determinar os valores das variáveis de decisão e, desta forma, as funções de utilidade de cada critério. Para fazer a implementação do método UTA, é necessária à matriz uma avaliação multicritério, isto é, a avaliação das alternativas em relação aos critérios. Para a pré-ordem $R$ definida em
$A$ ou $A^{\prime}$, em que $A^{\prime}$ é um subconjunto das alternativas representativas presentes em $A$, o agente decisor expressa sua preferência ou sua indiferença global para cada par $(a, b) \in A^{\prime}$.

Para cada alternativa de $A^{\prime}$, a função de utilidade calculada $U^{\prime}[g(a)]$ difere da verdadeira $U[g(a)]$ em um erro $\sigma(a)$ :

$$
U^{\prime}[g(a)]=\sum_{i=1}^{n} u_{i}\left[g_{i}(a)\right]+\sigma(a), \text { para todo } a \in A^{\prime}
$$

Considerando-se as relações de preferência (3) e de indiferença (4), além da função de utilidade (8), tem-se:

$$
\begin{gathered}
U^{\prime}[g(a)]-U^{\prime}[g(b)] \geq \delta \Leftrightarrow \\
\text { se o decisor indica } a P b \\
U^{\prime}[g(a)]-U^{\prime}[g(b)]=0 \Leftrightarrow
\end{gathered}
$$$$
\text { se o decisor indica } a I b
$$

sendo $\delta>0$ um número real pequeno empregado a fim de separar significativamente duas classes da pré-ordem $R$. Os autores do método UTA sugerem, na sua concepção original do método, que o valor de $\delta$ deve pertencer ao intervalo $[1 / 10 Q, 1 / Q]$, sendo $Q$ o número de classes de indiferença. Assumindo a existência de transitividade, o agente decisor só precisará fazer $(m-1)$ comparações entre alternativas, na quais $\mathrm{m}$ corresponde ao número de alternativas de $A^{\prime}$.

Substituindo (8) em (9) e (10), tem-se:

$$
\begin{aligned}
& \sum_{i=1}^{n}\left\{u_{i}\left[g_{i}(a)\right]-u_{i}\left[g_{i}(b)\right]\right\}+\sigma(a)-\sigma(b) \geq \delta \Leftrightarrow a P b \\
& \sum_{i=1}^{n}\left\{u_{i}\left[g_{i}(a)\right]-u_{i}\left[g_{i}(b)\right]\right\}+\sigma(a)-\sigma(b)=0 \Leftrightarrow a I b
\end{aligned}
$$

Consideram-se as funções $u_{i}$ como sendo lineares por intervalos. Para definir tais intervalos, o agente de decisão escolhe $\alpha_{i}$ pontos do intervalo $\left[g_{i *}, g_{i}^{*}\right]$ em que a função $u_{i}$ está definida. Determina-se cada ponto $g_{i}^{j}$ pela seguinte fórmula (Equação 13):

$$
g_{i}^{j}=g_{i^{*}}+\frac{j-1}{\alpha_{i}-1}\left(g_{i}^{*}-g_{i^{*}}\right)
$$

e $j$ variando de 1 até $\alpha_{i}$. Esses pontos vão definir $\left(\alpha_{i}-1\right)$ intervalos equidistantes de $\left[\mathrm{g}_{i *}, \mathrm{~g}_{i}{ }^{*}\right]$ (Equação 14).

$$
u_{i}\left[g_{i}(a)\right]=u_{i}\left(g_{i}^{j}\right)+\frac{g_{i}(a)-g_{i}^{j}}{g_{i}^{j+1}-g_{i}^{j}}\left[u_{i}\left(g_{i}^{j+1}-u_{i}\left(g_{i}^{j}\right)\right]\right.
$$

Satisfaz-se a hipótese da monotonicidade das preferências das utilidades marginais $u_{i}\left(g_{i}\right)$ requerida por este método pelo seguinte conjunto de restrições:

$$
\begin{gathered}
u_{i}\left(g_{i}^{j+1}\right)-u_{i}\left(g_{i}^{j}\right) \geq s_{i}, \mathrm{j}=1,2,3, \ldots,\left(\alpha_{\mathrm{i}-1}\right), \\
\mathrm{i}=1,2,3, \ldots, \mathrm{n} .
\end{gathered}
$$


em que $S_{i} \geq 0$ é o limite de indiferença definido para cada critério $i$.

Estimam-se as variáveis de decisão que representam as utilidades marginais $u_{i}\left(g_{i}^{j}\right)$ e os erros $\sigma(a)$ por meio do programa linear [PPL1] (16) considerando as seguintes restrições: restrições devido às preferências (11) e às indiferenças (12) entre as alternativas $a \in A^{\prime}$; restrições devido aos limites superiores (6) e inferiores (7) de cada critério; restrições que garantam a hipótese da monotonicidade das utilidades marginais (15); e restrições devido à não negatividade das variáveis de decisão do modelo, apresentadas nas inequações (17) e (18). A função objetivo do primeiro programa linear [PPL1] (16) corresponde à minimização do somatório dos erros $\sigma(a)$ associados às utilidades de cada alternativa $a \in A^{\prime}$, para respeitar a ordenação $a$ priori fornecida pelos decisores.

Sujeito a:

$$
\text { [PPL1] Min } F=\sum_{i=1}^{m} \sigma(a)
$$

$$
\begin{gathered}
\sum_{i=1}^{n}\left\{u_{i}\left[g_{i}(a)\right]-u_{i}\left[g_{i}(b)\right]\right\}+\sigma(a)-\sigma(b) \geq \delta \Leftrightarrow a P b \\
\sum_{i=1}^{n}\left\{u_{i}\left[g_{i}(a)\right]-u_{i}\left[g_{i}(b)\right]\right\}+\sigma(a)-\sigma(b)=0 \Leftrightarrow a I b \\
u_{i}\left(g_{i}^{j+1}\right)-u_{i}\left(g_{i}^{j}\right) \geq s_{i}, \mathrm{j}=1,2,3, \ldots,\left(\alpha_{\mathrm{i}-1}\right), \\
\mathrm{i}=1,2,3, \ldots, \mathrm{n} . \\
\sum_{i=1}^{n} u_{i}\left(g_{i}{ }^{*}\right)=1 \\
\mathrm{e} u_{i}\left(g_{i^{*}}\right)=0 \text { para todo } \mathrm{i} \\
u_{i}\left(g_{i}^{j}\right) \geq 0, \text { para todo i e j } \\
\sigma(a) \geq 0, \text { para todo } a \in A^{\prime}
\end{gathered}
$$

A solução ótima do primeiro programa linear [PPL1] (16) será aquela (ou aquelas) que obtenha o melhor valor para a função objetiva. Seja $F^{*}$ o valor ótimo obtido por meio do primeiro programa linear. O método UTA não para a obtenção de uma solução ótima do PPL1 (16), outras soluções são pesquisadas, incluindo-se,

$$
F \leq F^{*}+k\left(F^{*}\right)
$$

como uma nova restrição, em que $k$ é um valor real pequeno. Os vértices deste novo poliedro correspondem às funções de utilidade, em que um ou mais critérios atingem um peso extremo (ou máximo ou mínimo). Assim, a exploração do novo poliedro é feita resolvendo dois novos problemas de programação linear:

$$
\begin{aligned}
& \text { [PPL2] Min } u_{i}\left(g_{i}^{*}\right), \text { para } \mathrm{i}=1,2,3, \ldots, \mathrm{n} \\
& \text { [PPL3] Max } u_{i}\left(g_{i}^{*}\right), \text { para } \mathrm{i}=1,2,3, \ldots, \mathrm{n}
\end{aligned}
$$

para cada critério. Esta etapa é chamada de análise pós-otimização. Apresentam-se a seguir estes dois novos problemas de programação linear:

[PPL2] Min $u_{i}\left(g_{i}^{*}\right)$, para $\mathrm{i}=1,2,3, \ldots, \mathrm{n}$

Sujeito a:

$$
F \leq F^{*}+k\left(F^{*}\right)
$$

$$
\sum_{i=1}^{n}\left\{u_{i}\left[g_{i}(a)\right]-u_{i}\left[g_{i}(b)\right]\right\}+\sigma(a)-\sigma(b) \geq \delta \Leftrightarrow a P b
$$

$$
\sum_{i=1}^{n}\left\{u_{i}\left[g_{i}(a)\right]-u_{i}\left[g_{i}(b)\right]\right\}+\sigma(a)-\sigma(b)=0 \Leftrightarrow a I b
$$

$$
\begin{gathered}
u_{i}\left(g_{i}^{j+1}\right)-u_{i}\left(g_{i}^{j}\right) \geq s_{i}, \mathrm{j}=1,2,3, \ldots,\left(\alpha_{\mathrm{i}-1}\right), \\
\mathrm{i}=1,2,3, \ldots, \mathrm{n} \\
\sum_{i=1}^{n} u_{i}\left(g_{i}{ }^{*}\right)=1
\end{gathered}
$$

$$
u_{i}\left(g_{i^{*}}\right)=0 \text { para todo i }
$$

$$
u_{i}\left(g_{i}^{j}\right) \geq 0 \text {, para todo i e } \mathrm{j}
$$

$$
\sigma(a) \geq 0 \text {, para todo } a \in A^{\prime}
$$

$$
\text { [PPL3] Max } u_{i}\left(g_{i}^{*}\right), \text { para } \mathrm{i}=1,2,3, \ldots, \mathrm{n}
$$

Sujeito a:

$$
F \leq F^{*}+k\left(F^{*}\right)
$$

$$
\begin{gathered}
\sum_{i=1}^{n}\left\{u_{i}\left[g_{i}(a)\right]-u_{i}\left[g_{i}(b)\right]\right\}+\sigma(a)-\sigma(b) \geq \delta \Leftrightarrow a P b \\
\sum_{i=1}^{n}\left\{u_{i}\left[g_{i}(a)\right]-u_{i}\left[g_{i}(b)\right]\right\}+\sigma(a)-\sigma(b)=0 \Leftrightarrow a I b \\
u_{i}\left(g_{i}^{j+1}\right)-u_{i}\left(g_{i}^{j}\right) \geq s_{i}, \mathrm{j}=1,2,3, \ldots,\left(\alpha_{\mathrm{i}-1}\right), \\
\mathrm{i}=1,2,3, \ldots, \mathrm{n} \\
\sum_{i=1}^{n} u_{i}\left(g_{i}^{*}\right)=1 \\
u_{i}\left(g_{i^{*}}\right)=0 \text { para todo i }
\end{gathered}
$$




$$
\begin{aligned}
& u_{i}\left(g_{i}^{j}\right) \geq 0, \text { para todo i e j } \\
& \sigma(a) \geq 0, \text { para todo } a \in A^{\prime}
\end{aligned}
$$

O [PPL2] (20) pesquisa os valores mínimos que as variáveis que representam os valores máximos de cada critério podem assumir respeitando além das restrições do [PPL1] (16), a restrição (19) e a restrição referente à folga da solução do [PPL1] (16). Já o [PPL3] (21) pesquisa os valores máximos que as variáveis que representam os valores máximos de cada critério podem assumir respeitando as mesmas restrições do [PPL2] (20).

As variáveis de decisão deste modelo são as funções de utilidades marginais $u_{i}\left(g_{i}^{j}\right)$ dos critérios e o erro associado à ordenação das alternativas $\sigma(a)$.

Finalmente, pelo método UTA calcula-se as médias das variáveis que representam as funções de utilidades marginais $u_{i}\left(g_{i}^{j}\right)$ obtidas pela análise de pós-otimização [PPL2] e [PPL3]. Assim, por exemplo, em uma análise com seis critérios, doze implementações são necessárias, seis empregando o [PPL2] e seis empregando o [PPL3]. Com estas médias, pode-se avaliar a $U(g(a))$ de todas as alternativas $a \in A$, sejam ou não estas alternativas do conjunto de referência $A^{\prime}$.

\subsection{O Método Utilité Additive - CRiteria}

O método UTA-CR (RANGEL, 2002) busca obter funções de utilidades dos critérios que representem melhor as preferências dos decisores. Assim, partindo deste princípio, faz-se o emprego das preferências dos decisores não em relação ao conjunto das alternativas, mas sim em relação ao conjunto de critérios presentes na análise decisória. Deste modo, a ordenação a ser empregada como dado de entrada nesta variante UTA-CR será a ordenação das alternativas obtida com o auxílio de um outro método de agregação do Apoio Multicritério à Decisão.

Em um problema de Apoio Multicritério à Decisão, um conjunto de alternativas, que se apresentam como soluções deste problema, são avaliadas por um conjunto de critérios definidos por decisores. Normalmente, o número de alternativas é muito variável, um problema de decisão pode apresentar poucas alternativas viáveis ou inúmeras e, desta forma, fica difícil para os decisores expressarem suas preferências em relação ao conjunto, ou mesmo, a um subconjunto dessas alternativas (GOMES, 2007; GOMES; GOMES; ALMEIDA, 2006).

Já com relação ao conjunto de critérios de julgamentos das alternativas, busca-se sempre evitar um número de critérios superior a nove, partindo-se do estudo pioneiro de Miller (1956). A obediência a esse princípio torna mais fácil para os decisores a expressão das suas preferências em relação ao conjunto de critérios do que em relação ao conjunto de alternativas.
A variante UTA-CR, portanto, visa obter as funções de utilidades dos critérios tendo como referência às preferências dos decisores em relação ao conjunto de critérios, e não em relação ao conjunto de alternativas, como faz o método UTA. Desta forma, o método UTA-CR faz o uso de uma nova função objetivo com novos termos e novas restrições, além das utilizadas no [PPL1] (16). As funções de utilidade dos critérios presentes na análise obtidas por meio de método buscam expressar as preferências a priori fornecidas pelos decisores.

A Equação (22) apresenta a nova função objetivo do método UTA-CR.

Como se vê, a variante UTA-CR difere do método UTA em vários aspectos. O principal é que as funções de utilidade obtidas estão o mais próximo possível das preferências dos decisores. Um outro consiste na não necessidade de se fazer a análise pós-otimização quando se usa o método UTA-CR, uma vez que, em uma única implementação, obtêm-se os valores das variáveis de decisão.

$$
\operatorname{Min} F=\sum_{a \in A^{\prime}} \sigma(a)+\sum_{i=-1}^{n} \xi\left(u_{i-\mathrm{MIN}}^{*}\right)+\sum_{i=-1}^{n} \xi\left(u_{i-\mathrm{MAX}}^{*}\right)
$$

A função objetivo do problema de programação linear da variante UTA-CR (22) apresenta dois novos termos, além do termo que representa o somatório dos erros associados à utilidade global das alternativas: um representando o somatório do afastamento superior do peso sugerido pelos decisores e outro representando o somatório do afastamento inferior do peso sugerido pelos decisores, em que $\xi$ é um valor real muito pequeno.

O modelo busca determinar os valores mínimos das variáveis de decisão $u_{i-\text { MIN }}^{*}$ e $u_{i-\mathrm{MAX}}^{*}$, que estão associados aos pesos dos critérios $i$. Visando restringir ainda mais o conjunto de soluções viáveis, em relação ao método UTA, o método UTA-CR introduz duas novas restrições para as variáveis que representam o valor máximo de cada critério, uma buscando restringir este valor para mais (23) e outra para menos (24). Nas restrições (23) e (24), $w_{i}$ representa o peso atribuído ao critério $i$ pelos decisores.

$$
\begin{aligned}
& u_{i}\left(g_{i}^{*}\right)-\left(u_{i-\mathrm{MIN}}^{*}\right) \geq w_{i} \\
& u_{i}\left(g_{i}^{*}\right)+\left(u_{i-\mathrm{MAX}}^{*}\right) \leq w_{i}
\end{aligned}
$$

Portanto, o resultado desta variante do método UTA fornece funções de utilidade mais próximas das preferências dos decisores, pois os valores obtidos estão bem próximos dos pesos fornecidos aos critérios pelos decisores. $\mathrm{O}$ problema de programação linear apresentado abaixo [PPL4] (25) representa o modelo matemático do método UTA-CR, fazendo referência às preferências dos decisores em relação ao conjunto de critérios. 


$$
\begin{aligned}
& \text { [PPL4] Min } F=\sum_{a \in A^{\prime}} \sigma(a)+ \\
& +\sum_{i=-1}^{n} \xi\left(u_{i-\mathrm{MIN}}^{*}\right)+\sum_{i=-1}^{n} \xi\left(u_{i-\mathrm{MAX}}^{*}\right)
\end{aligned}
$$

Sujeito a:

$$
\begin{gathered}
\sum_{i=1}^{n}\left\{u_{i}\left[g_{i}(a)\right]-u_{i}\left[g_{i}(b)\right]\right\}+\sigma(a)-\sigma(b) \geq \delta \Leftrightarrow a P b \\
\sum_{i=1}^{n}\left\{u_{i}\left[g_{i}(a)\right]-u_{i}\left[g_{i}(b)\right]\right\}+\sigma(a)-\sigma(b)=0 \Leftrightarrow a I b \\
u_{i}\left(g_{i}^{j+1}\right)-u_{i}\left(g_{i}^{j}\right) \geq s_{i}, \mathrm{j}=1,2,3, \ldots,\left(\alpha_{\mathrm{i}-1}\right), \\
\mathrm{i}=1,2,3, \ldots, \mathrm{n} . \\
\sum_{i=1}^{n} u_{i}\left(g_{i}^{*}\right)=1 \\
u_{i}\left(g_{i^{*}}\right)=0 \text { para todo i } \\
u_{i}\left(g_{i}^{j}\right) \geq 0, \text { para todo i e j } \\
\sigma(a) \geq 0, \text { para todo } a \in A^{\prime} \\
u_{i}\left(g_{i}^{*}\right)-\left(u_{i-\text { MIN }}^{*}\right) \geq w_{i} \\
u_{i}\left(g_{i}^{*}\right)+\left(u_{i-\text { MAX }}^{*}\right) \leq w_{i}
\end{gathered}
$$

Diversas aplicações do método UTA-CR têm sido divulgadas por artigos (ARAYA et al., 2002; RANGEL, 2006; RANGEL et al., 2006; RANGEL et al., 2003).

\section{Estudo de caso}

Os dois métodos, UTA e UTA-CR, foram empregados para avaliar um conjunto de dez imóveis que estão disponíveis para serem alugados no município de Volta Redonda, no sul do Estado do Rio de Janeiro. Para realizar esta avaliação fez-se uma pesquisa junto às imobiliárias e especialistas do ramo, com a finalidade de identificar os critérios que deveriam ser considerados nesta análise e qual a importância de cada um desses critérios.

Não houve unanimidade nas informações coletadas das imobiliárias e do especialista. Trabalhou-se com um valor aproximado da média dos valores obtidos nessa coleta de dados. No total foram entrevistadas onze pessoas de seis diferentes imobiliárias, além do especialista.

\subsection{Definição dos critérios}

A partir dos pontos de vista explicitados nas entrevistas com profissionais do ramo imobiliário, identificaram-se os critérios mais relevantes para esta análise, descritos a seguir:

1) $C_{1}$ - localização:

É o mais importante critério de avaliação dos imóveis para aluguel. É um critério qualitativo que busca definir se o imóvel está ou não em uma localização bem valorizada. Para avaliar o desempenho das alternativas em relação a este critério, definiu-se a escala apresentada na Tabela 1. Desta forma, um imóvel com "boa localização", nesta avaliação, recebe uma pontuação igual a 4 .

2) $\mathrm{C}_{2}$ - área de construção:

Critério quantitativo que retrata o tamanho da construção do imóvel. Neste estudo, a unidade de medida utilizada foi o metro quadrado $\left(\mathrm{m}^{2}\right)$.

3) $\mathrm{C}_{3}$ - qualidade da construção:

Critério qualitativo que determina o padrão de acabamento do imóvel. Foram definidos três níveis de acabamento e suas respectivas pontuações, que são apresentados na Tabela 2. Considerando uma residência que tenha sido construída com um "alto padrão de acabamento", este imóvel recebe a pontuação igual a 3, valor máximo para este critério de avaliação.

4) $\mathrm{C}_{4}$ - estado de conservação:

Critério qualitativo utilizado para avaliar o estado geral do imóvel. Este critério considera se houve ou não reforma do imóvel e, também, suas condições de moradia. A Tabela 3, a seguir, apresenta a pontuação associada ao estado de conservação do imóvel. Por exemplo, um apartamento em condições de moradia, necessitando de pequenos reparos, ou seja, com "médio estado de conservação", recebe uma pontuação igual a 2. Este critério não está diretamente relacionado ao critério $\mathrm{C}_{3}$, pois o estado de conservação independe do padrão de acabamento do imóvel. Um imóvel pode ter um alto padrão de acabamento, com material

Tabela 1. Valoração do critério $C_{1}$ - localização.

\begin{tabular}{lc}
\hline \multicolumn{1}{c}{ Localização } & Pontuação Associada \\
\hline Periferia & 1 \\
Periferia/média localização & 2 \\
Média localização & 3 \\
Boa localização & 4 \\
Excelente localização & 5 \\
\hline
\end{tabular}

Tabela 2. Valoração do critério $\mathrm{C}_{3}$ - qualidade da construção.

\begin{tabular}{lc}
\hline \multicolumn{1}{c}{ Qualidade da construção } & Pontuação associada \\
\hline Baixo padrão de acabamento & 1 \\
Médio padrão de acabamento & 2 \\
Alto padrão de acabamento & 3 \\
\hline
\end{tabular}


de grande qualidade, mas estar precisando de reformas, devido à sua idade.

5) $\mathrm{C}_{5}$ - número de vagas na garagem:

Critério quantitativo e muito valorizado por pessoas que necessitam alugar um imóvel e possuem um ou mais veículos.

6) $\mathrm{C}_{6}$ - número de cômodos:

Critério quantitativo que considera para a valoração a quantidade dos seguintes cômodos existentes no imóvel: salas, quartos, copa/cozinha e banheiros. Destaca-se aqui a diferença entre este critério e o critério $\mathrm{C}_{2}$, que retrata a área de construção. É interessante avaliar o imóvel também pelo número de cômodos, pois podem estar em questão dois imóveis com o mesmo tamanho total, mas um deles pode ter poucos cômodos grandes, e o outro, com maior número de cômodos menores.

Os pesos atribuídos aos seis critérios, de acordo com as preferências dos decisores, são apresentados na Tabela 4. Estes valores dos pesos são apresentados na terceira coluna da Tabela 4 (Peso Atribuído) e representam a média aproximada dos valores fornecidos pelos decisores, que neste estudo de caso são as imobiliárias e o especialista.

Todos os critérios acima definidos são critérios de maximização, ou seja, quanto maior for a pontuação obtida na avaliação das alternativas em relação a cada critério, melhor será o seu desempenho.

\subsection{Definição das alternativas}

Dentre os imóveis disponíveis para serem alugados, os dez imóveis selecionados e os seus desempenhos em relação aos critérios presentes na análise são apresentados na Tabela 5 abaixo.

A ordenação das alternativas solicitada como dado de entrada, além da matriz de decisão apresentadas na Tabela 5, na implementação dos métodos UTA e UTA-CR, foi obtida com o emprego do método TODIM, e publicada por Rangel et al. (2006). Tal ordenação foi a seguinte: $A_{5}$, $\mathrm{A}_{1}, \mathrm{~A}_{4}, \mathrm{~A}_{8}, \mathrm{~A}_{3}, \mathrm{~A}_{2}, \mathrm{~A}_{6}, \mathrm{~A}_{10}, \mathrm{~A}_{9}$ e $\mathrm{A}_{7}$.

\subsection{Implementação}

Os métodos UTA e UTA-CR foram implementados pelo software LINDO (LINDO, 2007) e os resultados são apresentados por seis gráficos, um para cada critério, nos quais é possível verificar as diferenças dos resultados fornecidos pelos dois métodos, apresentados na Figura 1. Os pesos dos critérios considerados nas implementações dos dois métodos são os pesos normalizados dos critérios apresentados na última coluna da Tabela 4.

Os parâmetros definidos pelos decisores para realizar a implementação foram os seguintes: $\delta$ igual a 0,01 e $s_{\text {i }}$ igual a zero. Desta forma o parâmetro $\delta$ está dentro do limite sugerido por Jacquet-Lagrèze e Siskos (1982). Já o parâmetro $s_{\mathrm{i}}$ assumindo um valor nulo, significa que as funções de utilidades podem ser representadas por uma reta, se os resultados do modelo assim o permitirem.

Por intermédio das implementações dos dois métodos obtêm-se as funções de utilidades dos critérios pelo método UTA, representado por uma linha pontilhada, e pelo método UTA-CR, este representado por uma linha contínua. Os seis gráficos a seguir, apresentados na Figura 1, identificados para cada critério por meio de seus títulos, mostram as funções de utilidades determinadas pelos dois métodos para cada critério presente na análise.

\subsection{Análise de sensibilidade}

Depois de obtidos os resultados com a implementação dos dois métodos, foi realizada a análise de sensibilidade variando os valores dos parâmetros que são empregados no método UTA-CR para verificar a sua consistência. O valor de $\delta$ empregado na primeira implementação foi de 0,01 . Na primeira análise esse valor foi alterado para 0,001 e a implementação do [PPL4] (22) obteve como solução, o valor

Tabela 3. Valoração do critério $\mathrm{C}_{4}$ - estado de conservação.

\begin{tabular}{cc}
\hline Estado de conservação & Pontuação associada \\
\hline Ruim & 1 \\
Médio & 2 \\
Bom & 3 \\
Ótimo & 4 \\
\hline
\end{tabular}

Tabela 4. Atribuição de pesos para os critérios.

\begin{tabular}{clcc}
\hline Critério & \multicolumn{1}{c}{ Descrição } & $\begin{array}{c}\text { Peso } \\
\text { atribuído }\end{array}$ & $\begin{array}{c}\text { Peso } \\
\text { normalizado }\end{array}$ \\
\hline $\mathrm{C}_{1}$ & Localização & 5 & 0,294 \\
$\mathrm{C}_{2}$ & Área construída & 3 & 0,176 \\
$\mathrm{C}_{3}$ & Qualidade da construção & 2 & 0,117 \\
$\mathrm{C}_{4}$ & Estado de conservação & 4 & 0,235 \\
$\mathrm{C}_{5}$ & Número de vagas & 1 & 0,058 \\
& na garagem & & \\
$\mathrm{C}_{6}$ & Número de cômodos & 2 & 0,117 \\
\hline
\end{tabular}

Tabela 5. Desempenho e ordenação das alternativas.

\begin{tabular}{ccccccc}
\hline & $\mathbf{C}_{\mathbf{1}}$ & $\mathbf{C}_{\mathbf{2}}$ & $\mathbf{C}_{\mathbf{3}}$ & $\mathbf{C}_{\mathbf{4}}$ & $\mathbf{C}_{\mathbf{5}}$ & $\mathbf{C}_{\mathbf{6}}$ \\
\hline $\mathrm{A}_{1}$ & 3 & 290 & 3 & 3 & 1 & 6 \\
$\mathrm{~A}_{2}$ & 4 & 180 & 2 & 2 & 1 & 4 \\
$\mathrm{~A}_{3}$ & 3 & 347 & 1 & 2 & 2 & 5 \\
$\mathrm{~A}_{4}$ & 3 & 124 & 2 & 3 & 2 & 5 \\
$\mathrm{~A}_{5}$ & 5 & 360 & 3 & 4 & 4 & 9 \\
$\mathrm{~A}_{6}$ & 2 & 89 & 2 & 3 & 1 & 5 \\
$\mathrm{~A}_{7}$ & 1 & 85 & 1 & 1 & 1 & 4 \\
$\mathrm{~A}_{8}$ & 5 & 80 & 2 & 3 & 1 & 6 \\
$\mathrm{~A}_{9}$ & 2 & 121 & 2 & 3 & 0 & 6 \\
$\mathrm{~A}_{10}$ & 2 & 120 & 1 & 3 & 1 & 5 \\
\hline
\end{tabular}



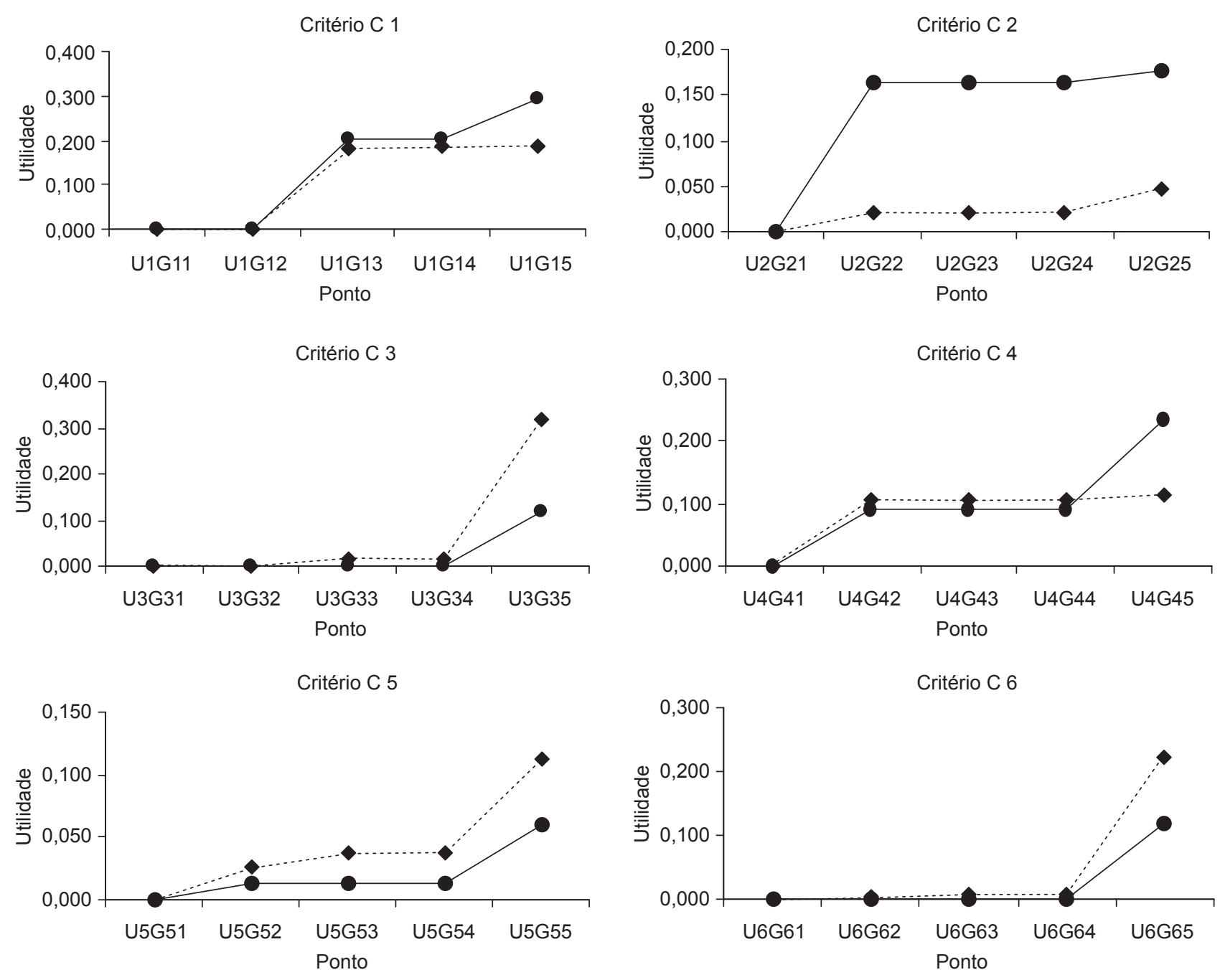

Figura 1. Funções de Utilidades dos Critérios $C_{i}(i=1,2, \ldots, 6)$.

nulo. Isto quer dizer que o somatório dos erros adicionados às utilidades globais das alternativas para representar as preferências dos decisores foi nulo, indicando que o modelo conseguiu representar as preferências sugeridas pelos decisores. Numa segunda análise o valor de $\delta$ foi alterado para 0,0001 e o resultado obtido foi o mesmo da implementação anterior, mostrando a consistência do método UTA-CR.

\section{Conclusão}

Verifica-se pelo exemplo implementado que os dois métodos apresentam valores bem diferentes para as funções de utilidades dos seis critérios empregados nesta análise. Os valores dos pesos dos critérios determinados pela variante UTA-CR estão bem mais próximos dos pesos fornecidos pelos decisores do que os determinados pelo método UTA.

Verificou-se também que a solução do [PPL1] (16) do método UTA apresentou um valor nulo. Isto que dizer que

o modelo matemático conseguiu obter a mesma ordenação proposta pelos decisores, mas este resultado pode não representar perfeitamente as preferências dos decisores, uma vez que a solução deste PPL é degenerada. Já o método UTA-CR impõe restrições aos pesos dos critérios, restringindo a região de busca das soluções ótimas. Um outro fato importante é que a função objetivo do método UTA-CR [PPL4] (25) busca obter valores para os pesos dos critérios com o menor afastamento possível dos valores dos pesos fornecidos pelos decisores, para mais ou para menos.

O método UTA-CR, por trabalhar com as preferências dos decisores em relação ao conjunto de critérios para, a partir daí, determinar uma ordenação das alternativas, obtém melhores formas das funções de utilidades. Vale ressaltar que estes resultados são obtidos por meio de uma única implementação do modelo matemático que representa este método, o que comprova a sua relevância dentre os poucos métodos do Apoio Multicritério à Decisão empregada na determinação das funções de utilidades. 
ASSOCIAÇÃO BRASILEIRA DE NORMAS TÉCNICAS - ABNT. NBR 14653-2. Norma brasileira para avaliação de imóveis urbanos. Rio de Janeiro, 2004.

ARAYA, M. C. G. et al. Building the additive utility functions for CAD-UFRJ. Annals of Operational Research, v. 116, n. 1-4, p. 271-288, 2002.

BELTON, V.; STEWART, T. J. Multiple CRiteria decision analysis: an integrated approach. Massachusetts: Kluwer Academic Publishers, 2002.

GOMES, L. F. A. M. Teoria da decisão. São Paulo: Pioneira Thomson Learning, 2007.

GOMES, L. F. A. M.; ARAYA, M. C. G.; CARIGNANO, C. Tomada de decisão em cenários complexos. São Paulo: Pioneira Thomson Learning, 2004.

GOMES, L. F. A. M.; LIMA, M. M. P. P. From modeling individual preferences to multicriteria ranking of discrete alternatives: a look at prospect theory and the additive difference model. Foundations of Computing and Decision Sciences, v. 17, n. 3, p. 171-184, 1992a.

GOMES, L. F. A. M.; LIMA, M. M. P. P. TODIM: basics and application to multicriteria ranking of projects with environmental impacts. Foundations of Computing and Decision Sciences, v. 16, n. 4, p. 113-127, 1992b.

GOMES, L. F. A. M.; GOMES, C. F. S.; ALMEIDA, A. T. Tomada de decisão gerencial: enfoque multicritério. 2 ed. São Paulo: Atlas, 2006.

GUIMARÃES NETO, C. A. Critérios de avaliação e NB502-89. In: Introdução a engenharia de avaliações e perícias judiciais. São Paulo: Ed. Instituto de Engenharia Legal, 1992. p. 45-60.

JACQUET-LAGREZE, E.; SISKOS, J. Assessing a set of additive utility functions for multicriteria decision-making the UTA method. European Journal of Operational Research, v. 10, n. 2, p. 151-164, 1982.

KEENEY, R. L.; RAIFFA, H. Decisions with multiple objectives: preferences and value tradeoffs. Cambridge: Cambridge University Press, 1993.
LINEAR INTERACTIVE AND DISCRETE OPTIMIZER - LINDO. Ilinois: Systems Inc. de Chicago, [200-]. Disponível em: <http:// www.lindo.com>. Acesso em: Março de 2007.

MILLER, G. A. The magical number seven, plus or minus two: some limits on our capacity for processing information. The Psychological Review, v. 63, n. 2, p. 81-97, 1956.

PESSÔA, M. Laudos técnicos, conceitos básicos e definições. In: Introdução a engenharia de avaliações e perícias judiciais. São Paulo: Ed. Instituto de Engenharia Legal, 1992. p. 1-26.

RANGEL, L. A. D. et al. A variant of the UTA method. In: INTERNATIONAL SYMPOSIUM ON MATHEMATICAL PROGRAMMING, 19, 2006, Rio de Janeiro. Anais... Rio de Janeiro: UFRJ, 2006. p. 91.

RANGEL, L. A. D. et al. Avaliação da interiorização dos cursos da UFF com o uso conjugado dos métodos UTA e MACBETH. Investigação Operacional, v. 23, n.1, p. 49-69, 2003.

RANGEL, L. A. D.; SILVA, S. S.; SILVERIO, L. B. Ordenação de imóveis residenciais empregando o método TODIM. In: SIMPÓSIO BRASILEIRO DE PESQUISA OPERACIONAL, 38, 2006, Goiás. Anais... Goiânia: SOBRAPO, 2006.

RANGEL, L. A. D. Determinações de funções de utilidade através das preferências dos decisores sobre o conjunto de critérios empregando o Método UTA. 2002. 154 f. Tese (Doutorado em Engenharia de Produção) - Universidade Federal do Rio de Janeiro, Rio de Janeiro.

RANGEL, L. A. D. Determinação de funções de utilidade dos critérios empregados na avaliação de imóveis residenciais. In: CONGRESO IBERO-LATINO AMERICANO DE INVESTIGACIÓN OPERATIVA, 13, 2006, Montevideo, Uruguai. Anais... Montevidéu: ALIO, 2006.

ROY, B.; BOUYSSOU, D. Aide multicritère à la décision: mèthodes et Cas. Paris: Ed. Economica, 1993.

VINCKE, Ph. L'aide multicritère à la décision. Bruxelas: Ed. de 1’Université de Bruxelles; Ed. Ellipses, 1989.

ZOPOUNIDIS, C.; DIMITRAS, A. I. Multicriteria decision aid methods for the prediction of business failure. Dordrecht: Kluwer Academic Publishers, 1998.

\section{Employing the Utilité Additive e Utilité Additive - CRiteria methods in real estate evaluation: a case study}

\section{Abstract}

This article presents an application of the UTA method and its variant UTA-CR to the determination of the utility functions for tackling the problem of evaluating residential real estate. Both methods belong to the American School of Multicriteria Decision Aiding. By using these methods, it is possible to determine the utility function of the CRiteria present in the evaluation analysis making use of previous information from the decision makers. The advantages of UTA-CR method over UTA are identified through the employment of the mathematical models embedded in each method. It can be observed that UTA-CR method makes use of decision-makers' preferences with respect to the CRiteria that lead to utility functions which are as close as possible to their preferences.

Keywords: UTA method. UTA-CR method. TODIM method. Multicriteria decision aiding. Multiattribute utility function. Residential real estate market. 


\section{Sobre os autores}

\section{Luís Alberto Duncan Rangel}

Escola de Engenharia Industrial Metalúrgica de Volta Redonda - UFF

Av. dos Trabalhadores 420, Vila Santa Cecília, CEP 27255-125, Volta Redonda, RJ

e-mail: duncan@metal.eeimvr.uff.br

\section{Luiz Flávio Autran Monteiro Gomes}

Ibmec/RJ

Av. Presidente Wilson, 118, Centro, CEP 20030-020, Rio de Janeiro, RJ

e-mail: autran@ibmecrj.br

Agradecimentos. Os autores gostariam de expressar sua gratidão aos revisores da versão original deste artigo. Graças aos seus comentários e sugestões aquela versão pode ser substancialmente melhorada. Os autores agradecem ainda ao CNPq a viabilização do Projeto de Pesquisa N.306658/2004-6.

Recebido: 14/3/2007

Aceito: $10 / 4 / 2009$ 